\title{
The PYTHEAS concept and applications
}

\author{
Y.P. Georgelin, G. Comte \\ Observatoire de Marseille, 2 pl. le Verrier, \\ 13248 Marseille Cedex, France \\ E. le Coarer \\ Observatoire de Grenoble, 414 rue de la Piscine, B.P. 53X, \\ 38041 Grenoble Cedex, France
}

\begin{abstract}
The PYTHEAS instrumental concept is an attempt to solve the problem of getting a complete tridimensional coverage of the observed field with a high spectral resolution across a wide spectral range. It is an integral field spectrometer derived from the TIGER spectrograph (field sampling with a microlens array) in which a scanning Fabry-Perot (FP) is introduced. The spectral resolution is given by the FP and the spectral range is that allowed by the grism of TIGER. The information is recorded on the CCD as a set of channeled spectra, for each microlens and each scanning step of the FP. The spectrum of each sampled field element is reconstructed from flux measurement of the Fabry spots defining the channeled spectra.

The application ranges of this instrument are reviewed with emphasis on the potential interest in globular cluster studies.
\end{abstract}

\section{Introduction}

Most existing spectro-imaging instruments, operating in the visible, face the "dimension curse" outlined by Monnet at the beginning of this Colloquium. When they provide high spectral resolution, either their spectral range or their field (or, more specifically, the number of spatially resolved elements) is limited to a narrow extent because of the finite size of the detector which must accomodate at the same time the two spatial dimensions of the field and the $\lambda$ dimension.

To achieve a high spectral resolution $(R \geq 10000)$ across a wide spectral range $(\delta \lambda \geq 1000 \AA)$ with a so-called "integral field" spectrographic device one obviously has to design a spectrometer at least up to the time when spectrally resolving detectors will become available. An elegant solution is provided in the near-infrared by the Imaging Fourier Transform Spectrometer (see Maillard et al., this Colloquium), thanks to the advent of 2D infrared detectors. We present here a different solution for the visible, based on the crossing of a Fabry-Perot interferometer with a grating. 


\section{A New Clone in Courtès' Toolbox}

\subsection{Principle}

The problem can be summarized as follows: how to get with the same instrument i) high spectral resolution, ii) wide spectral range, and iii) complete 2D spatial coverage of the field. Le Coarer and Connes (this Colloquium) presented the "ecological" solutions to preserve complete spatial sampling and spectral sampling in 3D spectroscopy.

i) The spectral resolution is provided by a Fabry-Perot interferometer (FP). It only depends on the finesse $F$ and the interference order $p$ and this latter can be very high if necessary. With $p=$ several hundreds and $F=20$ to 50, spectral resolutions $R=20000$ or more are easily achieved.

ii) The spectral range is best obtained with a low dispersion grating whose blaze function may be sufficiently soft across 2000 to 3000 A while keeping a very good light efficiency. A Fabry-Perot illuminated by a white light source acts as a spectral selector (a multi-monochromator) and gives a light modulated by the Airy function. The FP may be viewed as an "Airy comb" that filters the incident spectral energy distribution. If this resulting modulated light is dispersed on a detector, one gets what is usually called a channeled spectrum. Measuring the energy contained in each "channel" of known (through an adequate calibration) wavenumber of this channeled spectrum allows the reconstruction of the initial spectrum at the wavelengths transmitted by the FP. Scanning the FP across its complete free spectral range will therefore allow the complete reconstruction of the initial spectrum. Note that this principle had already been applied long ago with the S.I.M.A.C (Chabbal and Pelletier, 1964), in an infinetely small field, with a Lallemand electronic camera as detector. Unfortunately, this futurist instrument remained as a laboratory curiosity and was never applied to astronomy.

iii) Spatial sampling The problem is therefore to cross both dispersers in a single spectrometer, keeping the complete spatial sampling (i.e. a so-called integral field spectrometer), the grating becoming the interference order separator of the Fabry-Perot. The basic principle of the integral field spectrometer is to use an "explicit" field sampling (with a 2D coding device) that allows to separate field sampling from spectral sampling on the detector so that the spatial information does not mix with spectral information.

\subsection{Optical solution}

The optical solution (le Coarer et al. 1992) is provided by a modification of the TIGER integral field spectrograph (see e.g. Bacon, this Colloquium). Here, the spatial sampling is provided by a field-pupil transformation by means of a microlens array. A Queensgate Instruments scanning FP is introduced in front of the microlens array. Fig 1 shows the optical layout.

\section{Data acquisition and Spectrum reconstruction}

A complete description of the instrument, its operation and principle of data reduction is given in Le Coarer et al. 1995. 

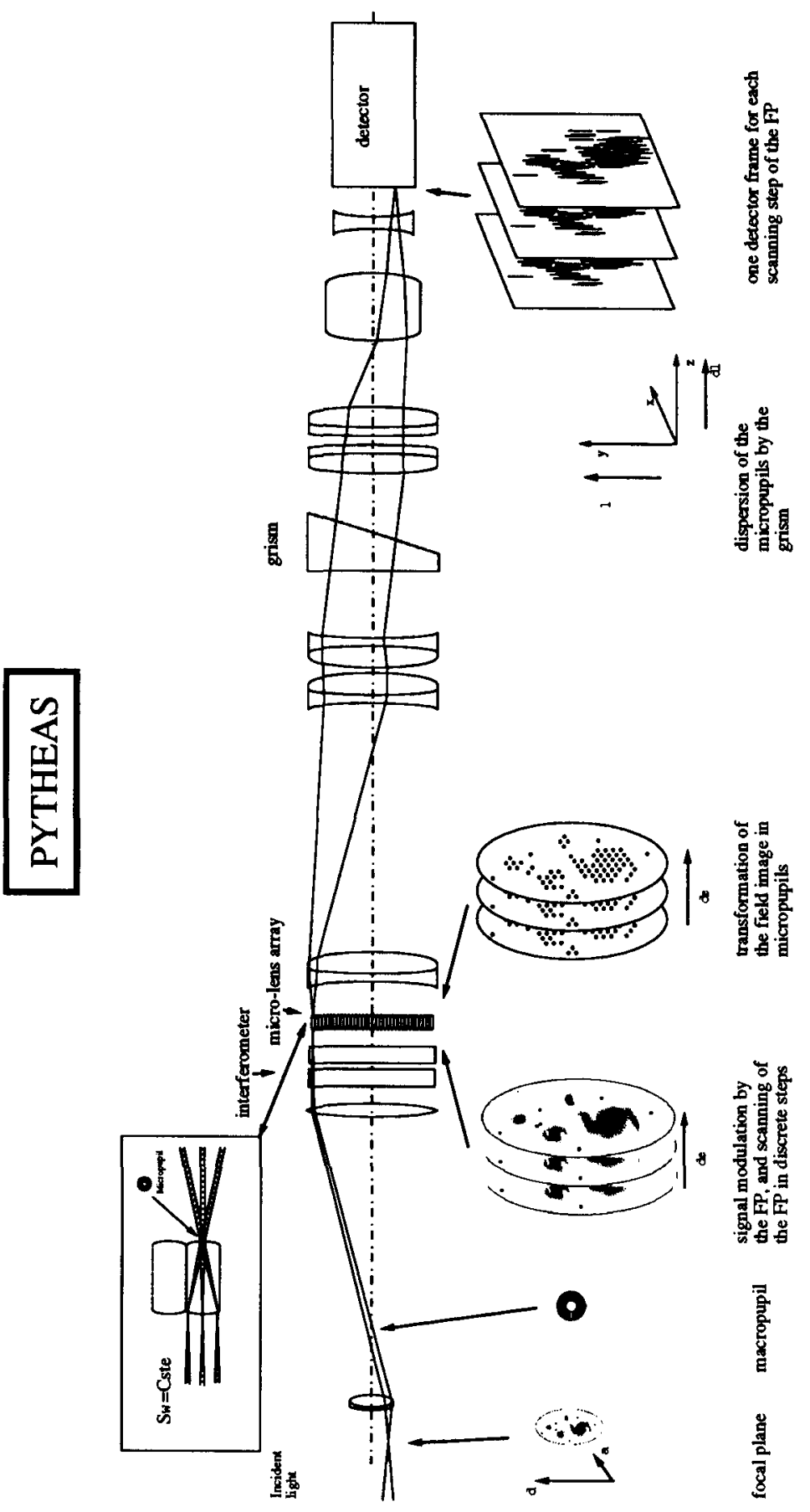

Figure 1. Optical layout of the PYTHEAS spectrometer 


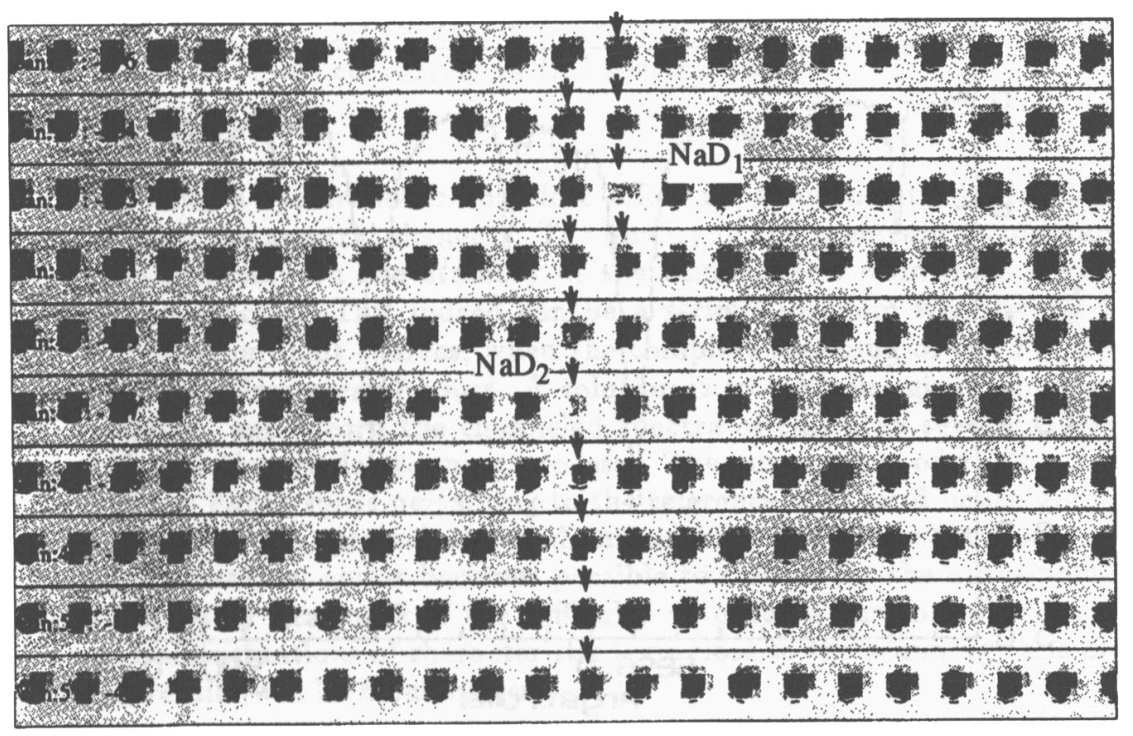

Figure 2. Channeled spectra of the Sun recorded with PYTHEAS. The figure is a montage of frames given by one single microlens, each horizontal row corresponds to a scanning step of the Fabry-Perot, so that along the vertical arrows, one follows contiguous resolved spectral elements appearing as Fabry spots. The channels associated with the $\mathrm{Na} I \mathrm{D}_{1}$ and $\mathrm{D}_{2}$ strong absorption lines give spots weaker than spots associated to neighbouring continuum

The data acquisition works as follows. For each scanning step of the FP, a channeled spectrum is recorded on the CCD. Any spectral feature, emission or absorption line, will give a brighter (emission) or fainter (absorption) Fabry spot on the detector, provided that its wavelength is transmitted by the FP at that step (Fig. 2). The scanning of the FP is synchronized with the readout of the CCD.

There is one independant channeled spectrum per micropupil, that is, per sampled field element. These channeled spectra appear as rows of "Fabry pupils" on the CCD. The wavenumber origin is determined by means of a calibration lamp exposure (channeled spectra of its emission lines). The wavenumber corresponding to each Fabry spot of each scanning step is therefore deduced. The flux in each spot is measured (using classical CCD photometry techniques, see e.g Gray in this Colloquium) and the spectrum given by each field element subsequently reconstructed. (see also Vlasiouk, this Colloquium, for the use of a photon-counting device as detector)

Ideally, the scanning procedure must sample exactly the free spectral range, that is, the number of scanning steps must be two times the value of the finesse. In case of undersampling, the reconstructed spectrum will suffer a degraded resolution. 


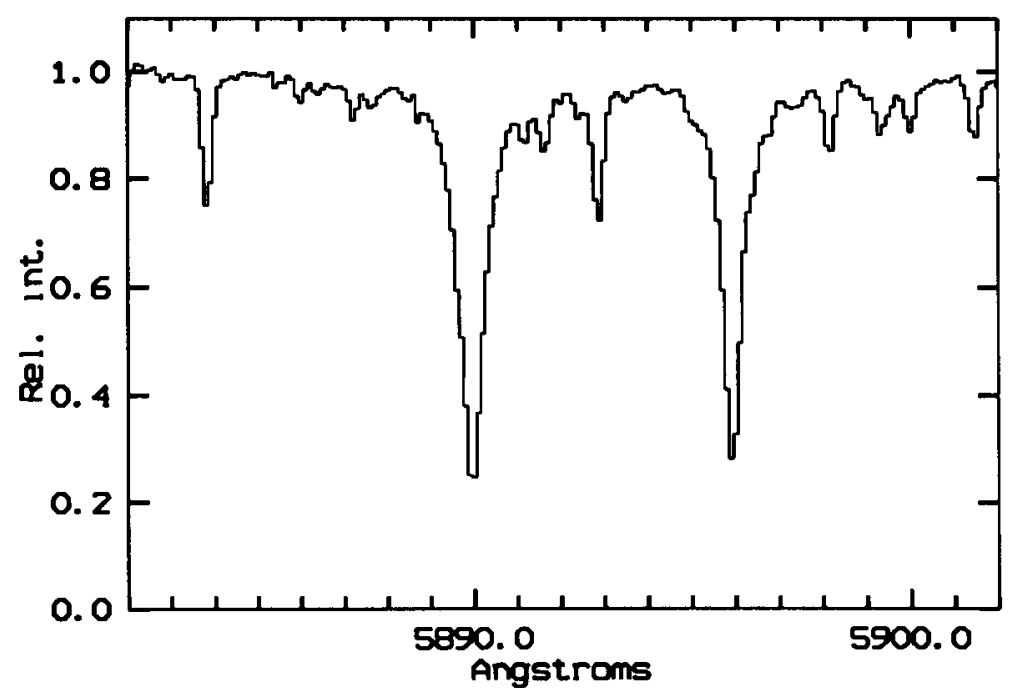

Figure 3. The reconstructed solar spectrum, taken on the Moon, in the vicinity of the $\mathrm{Na}$ I D lines. Weak features affecting the continuum are due to terrestrial water vapor

Figure 3 shows an example of the solar spectrum (taken on the full Moon at Observatoire de Haute-Provence) in a narrow window encompassing the $\mathrm{Na} \mathrm{I}$ $D$ lines, at a spectral resolution of about 19000 . Most of the weakest absorption lines are due to atmospheric water vapor. Measurements of wavelengths and equivalent widths of more than 100 unblended lines of moderate strength on this Moon spectrum have confirmed the excellent quality of the reconstructed data.

\section{Advantages and Drawbacks}

\subsection{Advantages}

PYTHEAS has all the merits of TIGER that are relevant to the complete 2D field sampling capability. It should specially be emphasized that it fully preserves the image quality of the (telescope + atmosphere) combination, because the spatial sampling is not provided by the detector, but by the microlens array, at the entrance of the system. Therefore, it can readily be designed to take the best of an adaptive optics corrugated focal plane.

The FP is used in extremely narrow beams of small spatial extent, hence in the optimal conditions of plate illumination. Under those conditions, the surface defects of the plates have only a very small effect on the finesse degradation. The resulting finesse is practically equal to the reflective finesse.

The optical layout is simple and compact and can be housed in reasonably sized dewars. Near infrared application of PYTHEAS may be envisioned as soon 
as sufficiently large-sized 2D IR detectors are available. Note however that the Imaging FTS is a serious concurrent in this spectral range.

\subsection{Drawbacks}

PYTHEAS being a spectrometer therefore offers no simultaneity in the data acquisition of all the spectral elements. The quality of the spectrum reconstruction depends critically on the stability of the atmospheric conditions during the acquisition process. This indeed can be a serious problem when working at high resolution on faint sources, when the complete scanning of the FP free spectral range requires several hours. A solution is to take one given scanning step as reference, and record the channeled spectra of this step several times, interspersed into the whole scanning process. The summation of all the flux contained in the Fabry spots pertaining to the reference step (and hence giving the same sample of the spectral energy distribution of the source) provides an internal self-calibration, and it becomes possible to correct for the atmospheric variations. Another drawback is the difficulty to observe variable objects: if quick temporal fluctuations of characteristic time close to the scanning time are present, the results will be meaningless.

\subsection{Performances}

With existing off-the-shelf components, the following performances are within reach on a $4 \mathrm{~m}$ - class telescope:

- spectral resolution : 6000 to 30000

- field : 7" x 7"

- spatial resolution : 0".5 (i.e. spatial element $0 " .25$ )

- sensitivity : at $R=20000$, with a $F P$ of finesse 25, for a 200 seconds exposure per scanning step on a CCD of $60 \%$ quantum efficiency and readout noise 6 electrons, a signal-to-noise ratio of 10 on the continuum of a V=16 star is expected.

\section{Astrophysical Applications}

\subsection{Potential topics}

PYTHEAS is well adapted to many astrophysical problems requiring a high spectral resolution coupled with a complete 2D field coverage, across a wide spectral range. A high spectral resolution is specially needed for line profile and line structure studies (in emission or absorption) and for high accuracy radial velocity determination. When available across of wide spectral range, cross-correlation methods become extremely powerful.

- Planetary surface studies : high resolution velocity studies of Jupiter, chemistry in the visible on Io, studies of cometary nuclei and comae.

- Circumstellar matter : environment of active stars (pre main-sequence stars, active supergiants) : physics of radiative transfer and fine velocity studies. Studies of mass loss. Ultracompact HII regions, bipolar flows, $\mathrm{HI} / \mathrm{HII} /$ molecular interfaces, Herbig-Haro objects. First trial observations at the Zelenchuk $6 \mathrm{~m}$ telescope (Vlasiuk, private communication) show 
the spectacular increase in information content when introducing a FP into the MultiPupil Spectrograph, transforming it into a PYTHEAS: a protostellar object observed in $\mathrm{H} \alpha$ line shows multiple velocity components at a spectral resolution of 9000 .

- Interstellar lines in stellar aggregates.

- Star clusters : globular clusters, compact open clusters (in nearby resolved galaxies), Trapezium systems.

- extragalactic objects : detailed physics in Active Galactic Nuclei and starburst nuclei, giant extragalactic HII regions and their ionizing clusters. Observations at the $6 \mathrm{~m}$ telescope of the nuclear region of the Seyfert galaxy NGC 1068 shows the multicomponent line structure of [Oiii]5007 as previously studied by Alloin and Pelat (1983). The spatial variations of the respective intensities of the components are easy to follow across the whole extent of the broad line region until its merging into the narrow line region, at a spatial resolution of 1 " (Vlasiuk, priv. comm.).

\subsection{Globular clusters}

Modern astrophysics on globular clusters requires a high spectral resolution, a capability to reach a substantial number of cluster stars in a given amount of telescope time, and, last but not least, an unambiguous identification of the targets, till, if possible, to the very center of the cluster. PYTHEAS is very well adapted to these challenges. Using cross-correlation techniques transforms PYTHEAS in a "multi-object" CORAVEL spectrometer (Baranne et al. 1979) and the expected performance is an accurracy better than $1.5 \mathrm{~km} / \mathrm{s}$ on $15 \mathrm{mag}$ nitude stars with a $4 \mathrm{~m}$ telescope. Thanks to the separation of spatial and spectral sampling in PYTHEAS, the field reconstruction and hence the target recognition will be limited only by the seeing quality.

These properties open various research domains, namely:

- dynamics of the stars, up to very small distance to the center of the cluster; search for ejected stars with anomalous velocities. (Meylan and Mayor 1991 and numerous references therein)

- search for binaries, by repeated observations of candidates, specially among blue stragglers. (Hut et al., 1992)

- seismology of RR Lyrae stars and BL Herculis stars.

- radial velocity distribution and velocity dispersion variation in light "cusps" of post-collapsed cluster cores; search for supermassive central object. (Peterson et al. 1989, Zaggia et al. 1992, Dubath et al. 1993)

- abundance studies on oxygen, carbon and various critical elements; studies of abundance heterogeneities in some clusters.

- search for intracluster interstellar matter from $\mathrm{Ca}$ II and $\mathrm{Na}$ I narrow absorption. 


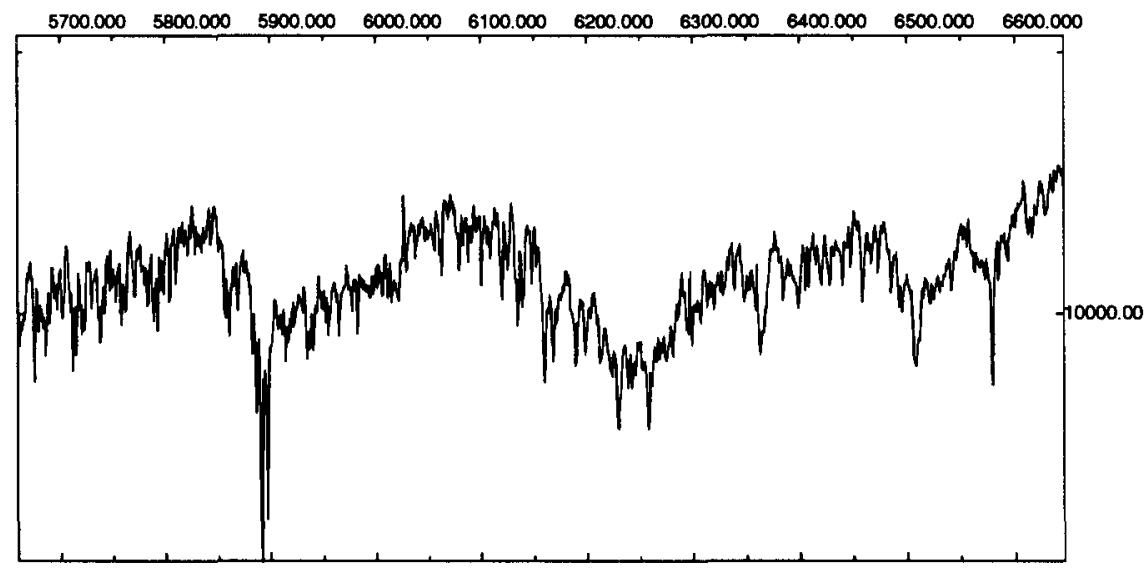

Figure 4. Spectrum of a star in the globular cluster $\omega$ Cen. The deepest absorption feature is due to the $\mathrm{NaI} \mathrm{D}$ lines (stellar and interstellar, partially superimposed)

- studies of $\mathrm{Ha}$ line profiles in asymptotic giant branch and red giant branch stars to revisit the very controversial mass loss problem.

Figure 4 shows a test spectrum on a star in $\omega$ Centauri taken at the ESO $3.6 \mathrm{~m}$ telescope with the first PYTHEAS-like prototype, housed in EFOSC-1, with a multihole mask as spatial coding device. 31 stars were simultaneously observed at a spectral resolution of $\mathbf{7 0 0 0}$ in a 4 hours scan. Although these results are still of poor quality (due to various problems, including poor atmospheric conditions), they are extremely encouraging for the future.

\section{References}

Alloin D., Pelat D., 1983, A\&A81, 172

Baranne A., Mayor M., Poncet J.L., 1979, Vistas in Astronomy 23, 279

Chabbal R., Pelletier R., 1964, Proc. of Conf. on Phot. and Spectro. Optics, Tokyo; J. Appl. Physics 4, Suppl. I, 445

Dubath P., Meylan G., Mayor M., 1994, A\&A, in prep.

Hut P., McMillan S., Goodman J., Mateo M., Phinney E.S., Pryor C., Richer H.P., Verbunt F., Weinberg M., 1992, P.A.S.P. 104, 981

Le Coarer E., 1992, Thesis, Université de Provence, Marseille

Le Coarer E., Bensammar S., Comte G., Georgelin Y.P., 1995, A\&AS(in press)

Meylan G., Mayor M., 1991, A\&A250, 113

Peterson R.C., Seitzer P., Cudworth K.M., 1989, ApJ347, 251

Zaggia S.R., Capaccioli M., Piotto G., Stiavelli M., 1992, A\&A258, 302 